Revue de l'Institut des langues et cultures

d'Europe, Amérique, Afrique, Asie et Australie

37 | 2019

Des genres en Méditerranée : pratiques,

représentations et transfert

\title{
Motifs en transhumance (Matisse et Ben Jelloun, ambassadeurs d'une esthétique méditerranéenne)
}

Transhumant Patterns (Matisse and Ben Jelloun, Ambassadors of a Mediterranean Aesthetics)

Nathalie Roelens

\section{OpenEdition}

Journals

Édition électronique

URL : http://journals.openedition.org/ilcea/8892

DOI : 10.4000/ilcea.8892

ISSN : 2101-0609

Éditeur

UGA Éditions/Université Grenoble Alpes

Édition imprimée

ISBN : 978-2-37747-099-0

ISSN : $1639-6073$

\section{Référence électronique}

Nathalie Roelens, «Motifs en transhumance (Matisse et Ben Jelloun, ambassadeurs d'une esthétique méditerranéenne) », ILCEA [En ligne], 37 | 2019, mis en ligne le 04 novembre 2019, consulté le 15 janvier 2020. URL : http://journals.openedition.org/ilcea/8892 ; DOI : 10.4000/ilcea.8892

Ce document a été généré automatiquement le 15 janvier 2020.

(C) ILCEA 


\title{
Motifs en transhumance (Matisse et Ben Jelloun, ambassadeurs d'une esthétique méditerranéenne)
}

\author{
Transhumant Patterns (Matisse and Ben Jelloun, Ambassadors of a
}

Mediterranean Aesthetics)

Nathalie Roelens

\section{Introduction : le syncrétisme}

Notre hypothèse d'un syncrétisme, voire d'un œcuménisme esthétique autour du bassin méditerranéen, exige quelques précisions notionnelles. L'acception du terme

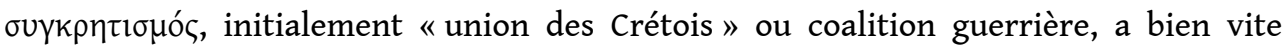
évolué vers l'idée de tentative de conciliation de différentes croyances, de rassemblement de doctrines disparates, et surtout de synthèse donnant lieu à des formes nouvelles. D'un point de vue épistémologique, on peut dès lors rapprocher le syncrétisme du bricolage au sens noble du terme que Claude Lévi-Strauss observait dans la pensée mythique amérindienne, à savoir le fait de créer à partir d'un répertoire limité de matériaux disparates un agencement nouveau engendrant une signification inédite (cf. Lévi-Strauss, 1962: 26). La synesthésie entendue comme entrée en connivence avec le monde, communion poétique, participerait-elle aussi de cette pensée syncrétique. Et Roland Barthes d'illustrer cette perte de sens au profit d'un gain d'atmosphère en comparant la lecture à une promenade dans un paysage marocain :

ce qu'il [le promeneur-lecteur] perçoit est multiple, irréductible, provenant de substances et de plans hétérogènes, décrochés: lumières, couleurs, végétations, chaleur, air, explosions ténues de bruits, minces cris d'oiseaux, voix d'enfants de l'autre côté de la vallée, passages, gestes, vêtements d'habitants tout près ou très loin; tous ces incidents sont à demi identifiables : ils proviennent de codes connus, mais leur combinatoire est unique, fonde la promenade en différence [...]. (Barthes, $1971: 75-76)$ 
2 De même, la stéréophonie de bruits, musiques et conversations qui environneront Barthes dans un bar de Tanger l'amèneront à réviser toute sa sémiologie à l'aune d'un syncrétisme sensoriel : «j'étais moi-même un lieu public, un souk; en moi passaient les mots, les menus syntagmes, les bouts de formules, et aucune phrase ne se formait, comme si c'eût été la loi de ce langage-là. » (Barthes, 1973 : 79) Ce vécu immersif ne se limite pas à la vue et à l'ouïe. Il est en quelque sorte responsable de la sublimation du goût physiologique en goût esthétique, en "sapience ", dont Anthelme Brillat-Savarin relevait les effets dans la luisance du visage après un bon repas: "Au physique, en même temps que le cerveau se rafraîchit, la physionomie s'épanouit, le coloris s'élève, les yeux brillent, une douce chaleur se répand dans tous les membres. Au moral, l'esprit s'aiguise, l'imagination s'échauffe, les bons mots naissent et circulent. » (BrillatSavarin, 1825: 112) C'est l'occasion pour nous de postuler une "sémiosphère » (Lotman, 1992) méditerranéenne, garante des systèmes de valeur et dépositaire de motifs païens, sacrés ou symboliques, autant d'arabesques et qui envahissent l'espace et enveloppent le spectateur dans leurs « divins méandres » (Aragon, 1926 : 209).

\section{Géo-esthétique}

3 Peintre novice depuis 2013 (après avoir consacré une Lettre à Matisse, 2013), pour se racheter de l'écriture - «Je ne me considère pas comme un peintre. Je suis un écrivain qui essaie de se racheter en faisant danser des couleurs prises dans le ciel et la mer de Tanger. » (Ben Jelloun, 2017 : 25) -, Tahar Ben Jelloun vient s'insérer dans une filiation d'arts décoratifs, se fait le relais d'une transmigration de motifs et de techniques qui irradient dans tous les domaines, faïences, mosaïques, tapisseries, zelliges. À en croire Abdelkébir Khatibi, la calligraphie s'imbrique dans l'architecture (mosquées, palais), dans les arts graphiques et ornementaux (miniatures, sculpture, mosaïque, verres émaillés) ou dans les arts usuels (céramique, tapis, tissus, bijoux, monnaies), manifestant partout la plénitude du sacré :

Ornementation bien connue : rosaces, fleurs, palmes, grappes, guirlandes... tout un enroulement de formes stylisées obéissant à des règles précises, et dont l'être est de manifester l'éclosion de l'omniprésence divine. Car le décor floral, par exemple, n'est pas comme on dit une pure abstraction. En tant que chose et chose épanouie, la flore retient, sous une vacuité apparente, le parfum de notre être. (Khatibi \& Sijelmassi, 1994 : 182-183)

Cette célébration du quotidien qui envahit l'espace, se tisse dans les objets, serpente le long des murs, nimbe le lieu d'une aura sacrée, traduit aussi une proximité avec le désir dans la mesure où les régimes totalitaires bannissent l'ornement comme déviant et sauvage. Pour Le Corbusier le progrès consistait précisément à s'en libérer : «Plus un peuple se cultive, plus le décor disparaît. » (Le Corbusier, 1996 : 85)

5 Aussi nous faut-il dresser une cartographie de la trajectoire de certains motifs ou stylèmes, telle une route de la soie ornementale. Henri Matisse, issu d'une famille de tisserands originaires de Bohain-en-Vermaindois, ville réputée pour ses luxueux tissus - sa mère modiste qui décorait des porcelaines a nourri son imagination et aiguisé son sens des couleurs -, suit en 1894 les cours de Gustave Moreau aux arts décoratifs de Paris, découvre les crêpons japonais au palais de l'Industrie et achète des tapis orientaux dans une boutique de Port-Royal (Millet, 2002: 15). En 1898, il quitte la grisaille du Nord à la recherche de la lumière du midi sur les traces de Van Gogh. En 
Corse "son art s'enflamme alors de l'incandescence de la lumière méditerranéenne, initiatrice de sa passion des couleurs " (Millet, 2002 : 9). Il récidive en 1904 à SaintTropez confectionnant des toiles de plus en plus éclatantes, ensoleillées, fougueuses, qui trouvent dans la polychromie un équivalent plastique à la représentation de la lumière. S'éloignant du divisionnisme de Signac, il peint en 1904 Luxe, calme et volupté et en $1905 \mathrm{La}$ joie de vivre, dont émane une sensualité mais aussi, par l'exaltation des couleurs et la négation de l'ombre, une véritable dimension spirituelle. L'été 1905 qui a donné lieu à La Vue de Collioure, signe dès lors à la fois l'activation de sa période fauve et le dépassement de celle-ci, car son esthétique abolit aussitôt de toute hiérarchie entre forme et couleur : « Le dessin et la couleur ne sont point distincts [...] Quand la couleur est à sa richesse, la forme est à sa plénitude » (Oursel, $1986: 11$ ). Cette dialectique entre dessin et couleur accompagnera toute la carrière de Matisse: "N'être que la limite d'une tâche de couleur, c'est trop peu pour la ligne. Elle a un droit total à l'indépendance. » (ibid. : 27)

Or, c'est grâce au Tapis rouge (1906), présenté au Salon de l'Industrie de 1906, qui intègre le personnage dans la surface décorative bidimensionnelle, en l'occurrence les tentures bigarrées, que Matisse sera accueilli en novateur en Russie. Sergei Ivanovitch Chtchoukine, lui-même collectionneur d'icônes de l'art russe et « importateur de tissus orientaux à Moscou » (ibid. : 15), demande au célèbre marchand Ambroise Vollard de lui présenter Matisse. Totalement subjugué, Chtchoukine commande pour sa maison de Moscou un panneau décoratif sur le thème de La Danse et La Musique que Matisse, qui n'aura aucun mal à s'incruster dans une esthétique tributaire de l'art byzantin, achèvera respectivement en 1906 et en 1910. Les personnages sont comme en apesanteur contemplative et invitent au recueillement. Pour la salle à manger du collectionneur russe, Matisse revisite radicalement sa nature morte La Desserte de 1897 en un ensemble vert, puis bleu et finalement rouge, La Desserte harmonie rouge (1908), où l'on assiste à une prise de possession de la toile par la couleur mais aussi par les formes car le nu emprunte les courbes du papier peint. De même, dans Nature morte en camaïu de bleu de 1909, l'on assiste à une symbiose entre vraies fleurs, fruits et ceux reproduits sur les tissus, proches de la très décorative toile de Jouy que Matisse collectionne, outre les châles espagnols et les faïences nasrides andalouses. Qui plus est, la grande exposition d'art islamique (entre autres des miniatures persanes) en 1910 à Munich cristallisera ses aspirations et lui révélera un espace plastique plus grand, donnant « dans un espace limité l'idée d'immensité » (Millet, 2002 : 45). L'horreur du vide propre à l'art islamique, l'espace bidimensionnel, le traitement du dessin par l'arabesque et la magie colorée de l'art oriental imprègnent désormais ses travaux.

7 Ce sont toutefois ses deux voyages initiatiques à Tanger en 1912 et 1913, d'une durée totale de sept mois, qui marqueront son esthétique. Depuis sa chambre d'hôtel (l'hôtel de France, chambre 35), qui a une vue sur la casbah et la baie de Tanger, il réalise ce que l'autre collectionneur d'art russe, Ivan Morozov, disposera de sa propre initiative en triptyque, à savoir Paysage vu de la fenêtre, Zohra sur la terrasse et La porte de la casbah. Ben Jelloun commente l'harmonie silencieuse que ces toiles communiquent: «On entend le silence. On sent le parfum du clou de girofle de la jeune femme » (Ben Jelloun, 2013 : 20) et souligne l'importance de ce séjour : «Après deux mois et demi à Tanger, vous repartez en France. Vous êtes changé. Apaisé mais habité par la Maroc, par sa lumière, ses parfums, par la gentillesse de ses habitants, par la diversité de ses couleurs. » (ibid.: 25) Le café arabe de 1912, espèce de café intérieur «à la limite de l'œuvre abstraite » transcende encore davantage tout souci anecdotique. Il présente au 
mur « un haïtté abstrait, sans broderies, sans fleurs, sans aucun dessin » (ibid : 28). Dans une lettre du 22 août 1912, Chtchoukine corrobore d'ailleurs l'universalité de ces motifs qui s'émancipent du figuratif: "Je pense beaucoup à votre tableau bleu (avec deux personnes), je le trouve comme un émail byzantin, d'une couleur tellement riche et profonde. » (Oursel, $1986: 14$ )

\section{Stylisation}

Le dessin et la couleur ne sont pas distincts pour Matisse mais évoluent tantôt vers la surenchère décorative et orientalisante comme dans Figure décorative sur fond ornemental de 1926 ou Odalisque à la culotte rouge de 1922, tantôt, à la fin de sa vie, vers des découpages qui lui permettent littéralement de dessiner, voire de sculpter la couleur, de faire surgir des formes qui s'apparentent à des feuilles, des palmes, des algues, des fleurs, des étoiles, des oiseaux, des coraux. Il va sans dire que cette plasticité du motif aura tout pour ravir Ben Jelloun :

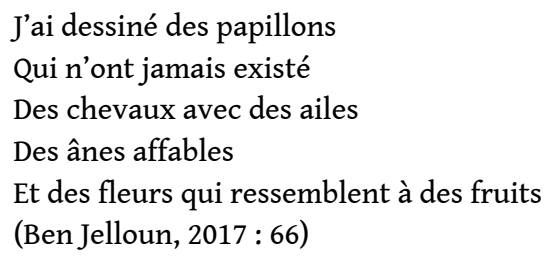

9 La question de savoir si une lecture symbolique de l'ornement est légitime est une question très répandue dans les débats esthétiques. À en croire Ernst Gombrich, le répertoire de motifs ou la stylisation en général émaneraient d'une crainte des populations primitives qu'une image ne prenne vie, croyance aux "pouvoirs surnaturels des symboles » (Gombrich, 1971: 148), pensée magique qui expliquerait pourquoi ils n'ont jamais voulu faire évoluer cette schématisation dans le sens d'une fidélité de représentation, d'une naturalisation. Khatibi ne dit pas autre chose. L'abstraction dans l'art islamique n'a rien d'un interdit de la figuration mais est " dirigé contre les survivances totémiques» (Khatibi \& Sijelmassi, 1994 : 128). Pour Georges Bataille, dans Lascaux ou la naissance de l'art, l'évacuation de la figure humaine de l'art rupestre témoigne d'une aspiration au jeu, à la part de de fantaisie ou de rêve que n'ont déterminés ni la faim ni le monde réel: «l'art pariétal ébranle le monde assujetti à l'utilité et régi par des valeurs qui refoulent la violence sacrée " (Bataille, 1980: 115). L'émergence de l'homme dans sa capacité à faire œuvre d'art, c'est-à-dire de créer des choses pérennes qui échappent à leur condition d'outil ou d'échange, va de pair, à en croire Bataille, avec l'émergence de l'art comme finalité sans fin, procès inaugural anthropo-historique d'institution de la culture. Il semble dès lors erroné de réduire à ce que certains sémioticiens appellent "signe plastique», en opposition à "signe iconique ", "les plombs des vitraux cisterciens, les entrelacs des enluminures irlandaises, les ouvrages de dames en macramé, etc. » (Groupe Mu, 1992 : 186) afin de cerner cette dimension « graphique » originaire du langage.

Les anthropologues et historiens de l'art, tels Jean-Claude Bonne, spécialiste du végétalisme dans l'art roman, voient en revanche dans la permanence de l'arabesque une pratique transculturelle et transhistorique bel et bien signifiante : « rien ne prouve que l'ornement soit dépourvu de sens, voire de plusieurs sens. Lui refuser une dimension symbolique équivaut à nier l'existence d'un imaginaire collectif chargé de mémoire générationnelle, de mythes et de croyances multiples, qu'il rend visible par la 
dimension irrationnelle qu'on lui attribue.» (Bonne, 2010: 31) L'apparente insignifiance peut donc être lourde d'un sens psychanalytique ou onirique, d'où «la proximité entre ornement et désir (d'un corps social ou d'un individu) et la part d'irrationnel qui impulse ses élaborations symboliques et ses mutations. » (ibid.: 37) Aussi conviendrait-il de parler non pas d'ornements aniconiques mais a-mimétiques, motifs floraux, végétaux, réseaux de feuilles stylisées. Plus les motifs ornementaux sont simples et séquentiels (spirales, damiers, ondulation), plus on les retrouve apparemment dans différentes cultures :

Ces motifs font sens au moins en tant que manière de s'approprier leur espace particulier et de le dynamiser en y introduisant de l'intelligibilité à la fois graphique et rythmique (comme les «pavages » d'un plan par des polyèdres ou les frises de rinceaux végétaux). Il ne s'agit pas d'un langage mais plutôt de schèmes sensibles suffisamment élémentaires pour être potentiellement universelles, susceptible d'être intégrés dans des dispositifs plus complexes à signification cosmologique, emblématique, morale, politique, magique ou autre. (ibid. : 33)

Khatibi met à l'épreuve du roman ce foisonnement décoratif, à savoir avec Le pèlerinage d'un artiste amoureux. Ce roman relate le cheminement de Raïssi, un stucateur d'arabesques, qui va jusqu'à se «mouler à l'esprit de la matière " (Khatibi, $2003: 320$ ). De surcroît, la tresse de plusieurs fils narratifs sinueux (les commandes d'art ornemental, l'amour charnel pour une femme sensuelle, le pèlerinage, le ciel et l'ange) noue et dénoue l'écriture, nous confrontant à un artefact hybride. Autrement dit, hanté par l'espacement lapidaire de son objet, le style de Khatibi épouse les courbes de la calligraphie arabe. Comme si son œuvre n'était lisible que lestée de sa charge décorative. En outre, certains passages épousent le graphisme spiralé de la calligraphie avec ses boucles, ses embrayages et débrayages, ses hampes et ses volutes :

Il m'arrivait d'accompagner mes lettres de quelques signes ornementaux. Motifs de zellige, de stuc, de géométrie ou de calligraphie. Des dessins et des graffitis, que la Sicilienne pouvait déchiffrer. Ces motifs visualisaient les traces de mon amour, ses rébus fous, nos rendez-vous imaginaires dans le labyrinthe. (ibid : 232)

12 Cet entrelacs narratif nous rappelle que la calligraphie relève toujours d'un « ensourcement miraculeux» (Khatibi \& Sijelmassi, $1994: 25)$, la traduction en image de l'incantation du Coran, la "transmigration de la voix divine dans le texte écrit." (ibid. : 29) La transfiguration du calligramme dans l'éros libérerait l'art de la théologie : "le verbe d'Allah arrive au chant intérieur, puis s'inscrit exotériquement dans le graphisme de l'écriture [...] lire est un éveil érotique sur la plage de l'être. » (ibid: 131) On peut donc relier la diversité des signes inscrits dans les corps (le tatouage au henné des femmes berbères) et les textes à la grande mystique islamique avec son idée de totalité environnante qui sature l'espace et enveloppe le sujet. Mais il nous faut rappeler que dans l'art ornemental le processus prime sur l'ouvrage achevé, entraînant «dans la danse » toute tentative herméneutique. À l'instar de "L'âme et la danse » (1921) de Paul Valéry, c'est le geste, en l'occurrence le tracé de la main dans la calligraphie, du pied chez Valéry, qui engendre le signe à interpréter. Peu importe si la danse exécutée par Athikte représente l'amour, la mer, la vie, l'acte pur des métamorphoses ou rien :

Elle semble d'abord, de ses pas pleins d'esprit, effacer de la terre toute fatigue, et toute sottise... Et voici qu'elle se fait une demeure un peu au-dessus des choses, et l'on dirait qu'elle s'arrange un nid dans ses bras blancs... Mais à présent, ne croiraiton pas qu'elle se tisse de ses pieds un tapis indéfinissable de sensations... Elle croise, elle décroise, elle trame la terre avec la durée... ô le charmant ouvrage, le travail 
très précieux de ses orteils intelligents qui attaquent, qui esquivent, qui nouent et

qui dénouent, qui se pourchassent, qui s'envolent !... (Valéry, 1924 :36-37)

\section{enfance lesquels se muent, dans son œuvre picturale primesautière, en un entrelacs} vibratoire de «lignes dansantes » ou de «traits chantants », tandis que l'artisanat, la céramique, le zellige et ses arabesques qui rayonnent partout lui dictent des stylèmes qu'il décline comme un alphabet magique: "C'est vrai que j'ai vu ma mère et ma grande sœur préparer le trousseau de mariage d'une parente et je me souviens d'elles à Fès brodant avec des fils mauve et bleu un tissu blanc cassé en coton assez épais, utilisé notamment pour les beaux coussins, les couvertures, les matelas. C'est un travail méticuleux. Il y avait également le hayti, issu de plusieurs couleurs, posé sur les murs et autour des portes. » (Ben Jelloun : 69) La verticalité de ces tapisseries semble en effet avoir déterminé les motifs verticaux récurrents de ses acryliques : la porte, le paravent, le marabout, les tables de la loi. D'ailleurs, dans l'échange fantasmé que constitue la Lettre à Matisse, Ben Jelloun projetait déjà sur le peintre sa fascination pour cet artisanat de luxe: "Une broderie de Fès était pour vous un enchantement parce que vous y découvriez tout le temps de nouvelles formes et perspectives. Une multitude de motifs, des arabesques, une géométrie non rigoureuse, une échappée de tous les songes » (Ben Jelloun, 2013: 16). Là encore, le fait qu'il n'y ait pas la moindre concession à l'anecdotique ne veut pas dire que le sens soit aboli mais que celui-ci relève d'une dimension cosmique, sacrée qui anoblit l'objet usuel et le dote d'une émotion esthétique : "C'est un art qui célèbre le quotidien des bijoux. Une révélation. Vous constatiez combien un tapis est une œuvre d'art (utilitaire), avec des signes, des métaphores florales, des losanges, des étoiles tissées comme s'il fallait refléter le ciel. Cette profusion de couleurs et de formes vous enchantait » (ibid.).

\section{Vocalisation / Colorisation}

Dans la calligraphie arabe la voyelle confère une couleur à la consonne atone selon un agencement entre ligne consonantique, "squelette littéral» ou "cadavre», et la partition de voyelles, floraisons curvilignes, vibratoires, «âme vivifiante " (Khatibi \& Sijelmassi, 1994 : 37), le tout formant « une scène pluridimensionnelle dérivée et nouée en un temps pulsatif " (ibid.: 49), la hampe de la scriptio consonantique se voyant complétée par le triangle vocalique de la scriptio plena. De façon analogue, dans une perspective d'œcuménisme plastique, les mosaïques byzantines ne seraient rien sans leur façon de refléter la lumière grâce à la technique d'incliner légèrement les tesselles par rapport au plan de pose créant une sensation de vibration de la surface, technique qui a ensuite influencé le royaume normand de Sicile mais aussi les artistes musulmans pour la décoration des mosquées et des palais.

L'exposition à l'Institut du Monde arabe de Paris (2017) montre bien que cette synergie entre arts appliqués, calligraphie arabe et mosaïques byzantines et en outre le miroitement polychrome avec lequel Matisse a dépassé le fauvisme sont un ensemble de déclencheurs pour Ben Jelloun trop enchaîné à une parole, certes poétique, mais toujours langagière.

Depuis que je peins de grandes toiles, je tiens à y déposer quelques phrases, des vers, des pensées. Une façon de ne pas oublier d'où je viens. J'écris aussi sur le cadre, entourant ainsi la toile de poésie avec plusieurs couleurs. Le texte n'est pas

ILCEA, 37 | 2019 
un commentaire de la toile. Il est indépendant, une autre célébration de la lumière

et de la joie. (Ben Jelloun, $2017: 20$ ) mars à mai 1990 à faire de la fiction à partir de la détresse de la réalité locale, découvre la ville troglodytique de Matera en Basilicate : «Je ne savais pas que la vieille ville de Fès [...] avait une sœur jumelle en Italie, à l'ouest de Bari. Elle s'appelle Matera » (ibid : 93). Inscrite au patrimoine mondial de l'Unesco, «deuxième Bethléem », ville la plus ancienne après Alep et Jéricho, elle incarne s'il en est le syncrétisme au sens religieux cette fois : païenne, avec ses habitations paléolithiques dans des grottes naturelles qui ont abrité des lieux de culte rupestres ; chrétienne ( « c'est probablement dans la grotte des Cent Saints que Dieu a séparé la lumière des ténèbres " (ibid: 94$)$ et aux VII ${ }^{\mathrm{e}}$ et VIII ${ }^{\mathrm{e}}$ siècles, les grottes devinrent le refuge de moines byzantins qui transformèrent leurs murs en chapelles ; arabe, en 856 les Arabes venus de Bari ont occupé Matera quelques jours ; touristique... L'insoutenable fouillis de maisons emboîtées de la ville troglodyte lui rappelle sa Fès natale, ville elle-même juive, arabe, berbère. Or, tout comme Matisse qui fait « chanter les couleurs » (Millet 2002 : 12), l'auteur marocain a ressenti le besoin de les dessiner dans un foisonnement de couleurs qui justement manquent au réel: «J'ai eu envie d'habiller cette ville unique de rêves et couleurs parfois vives, de couleurs imaginées, posées là comme une robe sur les épaules de la mariée, une robe ou une broderie du xix ${ }^{e}$ siècle tissée par les mains de femmes de Fès avec des fils d'or acheté au mellah, quartier juif à la sortie de la médina. Cette robe couvre les terrasses quand la nuit arrive pleine de rêves impatients, de jarres de miel et d'huile d'olive.» (Ben Jelloun, 2017 : 93) Cette isotopie se décline à plusieurs reprises : «Je l'ai peinte comme une ville rêvée [...] C'est une Matera de fête. Des couleurs sont venues l'habiller comme une mariée marocaine" (ibid: 97). L'image surgissait déjà dans sa prose, notamment dans sa nouvelle italienne "Nuit africaine ", où Ben Jelloun campe un Napolitain (Liternois) qui fait le vœu d'avoir cent ans à Tanger, ville qu'il imagine " comme une superbe mariée, toute de lumière, de perles, de diamants et de magie " (Ben Jelloun, 1992: 81). Cette volonté de coloriser relève-t-elle aussi d'un invariant méditerranéen ou d'un tropisme humain?

Sans prétendre épuiser la question, on pourrait invoquer l'éthologie pragmatique du biologiste Jakob von Uexküll qui, dans sa Umweltlehre (1956) (théorie des milieux, qui deviendra la mésologie chez Augustin Berque), recourt à la métaphore musicale du Ton, au sens de disposition du sujet (tonalité affective) : «Dans tous les objets dont nous avons appris l'usage, nous voyons la performance que nous accomplissons grâce à eux, avec la même certitude que leur forme ou leur couleur " (Von Uexkull, 2010: 109), Schutzton, «tonalité de défense », Wohnton, « tonalité de résidence », Esston « tonalité de nutrition ». Uexküll passe ensuite d'une métaphore musicale, la tonalité (Ton), à une métaphore picturale, la coloration (Tönung), avec la même idée de rayonner sur son environnement pour le transformer en milieu. Le Sitzton devient Sitztönung, coloration de s'asseoir. Si le mobilier dans une pièce possède pour l'humain des colorations de nourriture, de s'asseoir, de lecture, d'éclairage, de déambulation, pour le chien elle aura seulement une coloration de nourriture et de s'asseoir. Tout le reste présente une coloration d'obstacle. La mouche s'intéressera juste à la lumière et à la chaleur. Une vache interprète l'herbe comme nourriture tandis que le chien lui trouve une autre fonction, de sorte qu'ils ne vivent pas dans le même milieu même s'ils partagent objectivement le même environnement. 

sémiosphère méridionale, où la nature luxuriante s'offre comme exemple à égaler et incite les artistes et artisans à exacerber les tons. Une autre généalogie s'impose alors. Matisse, comme pour chercher un étayage théorique à son engouement pour les couleurs du sud, s'intéresse à la théorie des couleurs du chimiste Eugène Chevreul. Celui-ci, dans sa qualité de directeur de la manufacture des Gobelins, avait été confronté à la demande des teinturiers qui cherchaient à améliorer le rendu des pigments utilisés. Ses expérimentations ont abouti à la loi du contraste simultané, à savoir le fait que lorsque l'œil perçoit en même temps deux couleurs avoisinantes, elles paraissent dissemblables, tant du point de vue de la composition optique que de leur valeur tonale. Le titre de son mémoire de 750 pages de 1839 est éloquent eu égard à la variété d'applications possibles de sa théorie : De la loi du contraste simultané des couleurs et de l'assortiment des objets colorés considérées d'après cette loi dans ses rapports avec la peinture, les tapisseries des Gobelins, les tapisseries de Beauvais pour meubles, les tapis, la mosaïque, les vitraux colorés, l'impression des étoffes, l'imprimerie, l'enluminure, la décoration des édifices l'habillement et l'horticultures. Il y montre par exemple qu'un même ton semble plus clair s'il est sur un fond plus sombre, mais surtout que les complémentaires se rendent plus vives mutuellement. Ainsi, un jaune placé près d'un vert semble plus rouge, comme si on l'avait mélangé à un pourpre, complémentaire du vert, tandis que la même teinte, placée près d'un rouge, tirera vers le jaune-vert. Cette théorie des couleurs permettra donc à Matisse et, partant, à Ben Jelloun d'exploiter pleinement les effets chromatiques et intensifs de leur palette.

L'on observe en outre que les cultures méditerranéennes adoptent cette exacerbation par contraste non seulement dans le visuel mais aussi dans les saveurs. Ainsi, la caponata, plat diffusé dans tout le monde méditerranéen, notamment en Sicile, à Naples et en Tunisie, est une espèce de ratatouille mais aigre-douce, car vinaigrée et comprenant des courgettes et des câpres, généralement consommée froide, après avoir délivré tous ses arômes. Ce qui rejoint notre définition initiale de syncrétisme se retrouve dans le fait que le disjoint des saveurs composites persiste malgré le mélange, le conjoint. Les papilles gustatives distinguent amertume, douceur, acidité, aigreur dans un tout supérieur à la somme de ses parties tout en s'enrichissant du contraste. On pourrait illustrer ce phénomène du discret dans le continu par un tableau emblématique de Renato Guttuso, La Vucciria de 1974, littéralement « la boucherie » qui figure le marché éponyme à Palerme, haut en couleurs et en saveurs, par une disposition verticale en mosaïque de tons vifs et de silhouettes hyperréalistes noyées dans les denrées fraîches. Une autre illustration serait Le souk de Tanger de Ben Jelloun (2013) qui empile jusqu'à saturation des formes colorées sur des vagues esquissées d'un trait naïf.

Cette véritable sémiosphère méditerranéenne, qui englobe les formes, les couleurs, les goûts se distingue, comme nous l'annoncions, par son acuité intensive. Elle s'oppose par sa sapidité, son "esculescence» (Brillat Savarin parle de "corps esculent» - comestible et sapide -, Brillat-Savarin, $1825: 56$ ), au degré zéro du goût et dès lors des autres sensations: la blancheur, l'inodore, le mou, le pâteux, l'incolore, le translucide, le silencieux. L'Amérique est pour Michel Serres ce zéro de la sapience et de la sagacité, l'incarnation de la frigidité sensorielle. Les Américains (protestants, puritains, végétariens) s'abreuvent littéralement de fadeur :

La moutarde, faible, n'a pas de goût; la bière, presque sans alcool, a perdu toute saveur, molles les épices, léger le café, à peine grillé, monotones les fruits et 
légumes, jusqu'à l'indifférencié. [...] le vin se change en lait, blanc. Rien ne pique ni ne blesse. L'Amérique mange mou. Et boit fade, bouche plate. Glacé de surcroît pour engourdir les papilles. [...] La mollesse alors flotte à l'entour des corps gloutons, l'homo insipiens se dessine à contours flous, gonfle et se fait monstre, perd ses formes, non pas gros, mais enveloppé dans la grossesse, redevenu embryon. Le commun dénominateur, unité monotone, arase les épines, rabote les épices, adoucit, amollit, annule odeurs et goûts. L'Amérique vit en paix. [...] Le surgelé inodore pour obèse cotonneux, dissimulé sous cellophane pour que nul ne goûte ni ne le touche. » (Serres, 1985 : 201-202)

21 C'était déjà l'étonnement du Barthes des Mythologies devant l'engouement des Américains pour le lait: "gage d'une force non révulsive, non congestive mais calme, blanche, lucide, tout égale au réel. » (Barthes, 1957 : 80) Il conviendrait d'opposer dans ces conditions les cultures de l'anesthésie à celles de l'hyperesthésie (l'Orient, le Mexique) et entre les deux : le bassin méditerranéen dont les saveurs déjà plus relevées entraînent des couleurs vives et des odeurs capiteuses. Ou encore, dresser des cartes d'ethnologie sensorielle: le palais au beurre et la bouche à l'huile qui se partagent l'Europe, les couleurs chamarrées flottant sur les corps dans le sud, la brume qui ternit et assombrit le vêtement et le teint dans le nord, une partition architecturale entre le rectiligne et l'ondoyant, la norme et le dé-viant, etc.

\section{Rythmesthésique}

Il nous faut toutefois revenir à la dialectique entre stylisation et chromatisme et proposer l'hypothèse qu'elle puisse se résoudre par le concept de «rythme». La puissance de l'art ornemental réside selon Khatibi dans la variation qui affecte l'invariant du motif. L'arabesque « porte l'équilibre des lignes et couleurs à leur point de vacillement par un entrelacs vibratoire entre l'épigraphie et le décor floral ou géométrique " (Khatibi \& Sijelmassi, 1994 : 170). Dans le sillage de L'esprit des formes d'Elie Faure, pour qui le rythme n'est pas seulement temporalité mais spatialité, le philosophe phénoménologue Henri Maldiney souligne à son tour l'élan vital qui jaillit du mouvement giratoire et de la dilatation, qui dépassent toute figuration au profit des formes voire de la formation, tels les «tourbillons solaires" (Maldiney, 2012 : 154) des Tournesols de Van Gogh, la Gestalt au profit de la Gestaltung. Maldiney conçoit le rythme comme « surgissement », « apparition » (ibid. : 154), après « écroulement de la figure du monde » (ibid.: 153). Il prend pour exemple la sobriété figurative des Sainte-Victoire de Cézanne (1904-1906), non moins tourbillonnantes, vertigineuses, par rapport aux tableaux qui représentent la même montagne du XVII ${ }^{\mathrm{e}}$ au XIX ${ }^{\mathrm{e}}$ siècle. Pour Maldiney, il y a quelque chose dans le rythme qui lui donne un caractère absolument fluide, au-delà de la forme. L'un des paradoxes notables du rythme serait alors que, tout en étant constitué d'éléments que l'on peut analyser comme étant discontinus, il se donne toujours à nous sous la forme synthétique d'une continuité - et d'une continuité en mouvement. Le rythme est proche du souffle, de la pulsation, de la modulation. Maldiney s'intéresse dès lors à l'auto-mouvement du rythme, aux altérations chromatiques et à l'autogenèse de la forme, « un monde qui n'a pas encore cristallisé en objets » (ibid.: 191). Et Cézanne de venir encore à son secours : « Regarde! Les bleus ! Les bleus là-bas sous les pins!» Mais aussi « Nous germinons» (ibid.: 204). Cézanne n'admet d'éléments que le peu qui suffit à mobiliser une surface "en nappe énergétique, en énergie spatialisante " (ibid. : 223). Cette énergie est fonction - et l'on retrouve ici la théorie de Chevreul - de la discontinuité des rencontres entre les tons, 
qui sont comme des "phonèmes" de la langue : "Plus ils sont différents, et même contraires, plus l'œuvre gagne en acuité » (ibid.: 226). Mais tandis que Cézanne atteint un certain équilibre, "une immobilité tendue dans laquelle le monde entier est en suspens" (ibid. : 251), le rythme "vit» et, sous peine de s'anéantir, doit sans cesse rejaillir. Les enjeux de cette "rythmesthétique » sont importants. Non seulement, le rythme serait commun à tous les arts, transartistique, mais l'œuvre rythmique serait celle qui parviendrait à retrouver quelque chose d'originaire dans notre rapport au monde, selon l'étymologie de rhuthmos comme configuration particulière du mouvant. De sorte que «la perception d'un rythme est en réalité une rythmisation de la perception. Et ce qui se joue dans le rythme, c'est à la fois la position du sujet et son déplacement, son décentrement.» (ibid.: 229) Cet élan cinétique se traduit chez Ben Jelloun par les couleurs et les lignes qui, dans l'espace totalement saturé de formes, semblent entamer un dialogue serré entre elles, se rapprocher et s'éloigner au gré du mouvement rapide, allegro, vivace, ou lento, adagio, selon que la trame est plus ou moins raréfiée.

Forte de cette découverte que l'art décoratif opère la jonction du tracé et de la couleur dans un ensemble rythmique, nous pouvons rapprocher le motif aussi de ce que JeanFrançois Lyotard (1972) ou Gilles Deleuze (1984) ont qualifié respectivement de Figure comme puissance figurale libidinale ou comme sensation, à même de dépasser ou de déconstruire la figuration (c'est-à-dire à la fois l'illustratif, le narratif, le lisible). Libérer la figure de la suprématie de la historia au sens albertien du terme qui engage un travail d'exégèse, équivaut en revanche à accepter son opacité et l'irréductibilité du visible au lisible. Il ne faut toutefois pas voir ce mouvement engagé contre la « ratio occidentale qui tue l'art en même temps que le rêve " (Lyotard, 1972:14) comme une manière de prôner quelque formalisme gratuit, un jeu asignifiant de la figure. Autrement dit, le figural ou la pure figure fait sens sans faire histoire en dehors de l'ordre discursif. Le figural se veut bien plutôt expression d'une réalité en excès, en débordement sur l'ordre discursif et intelligible. La question du sens est moins évacuée ou mise hors circuit que profondément modifiée: l'espace figural exprimerait et désignerait l'événement a-logique et a-discursif par excellence du désir. Il y a, écrit Lyotard, une « connivence du désir avec le figural» (ibid. : 271) qui aboutit à la «figure-matrice»: espace de transgression de toute logique et de tout logocentrisme, espace de non-dit, mais aussi force vitale que Deleuze repère chez Francis Bacon, force dynamique rythmique et pulsionnelle, défigurante. Or, ce qui se passe en aval chez Lyotard ou Deleuze, dans l'après-coup du figuratif, se déploie en amont et se pérennise à toutes les époques dans la fougue ornementale. Ce qui a eu besoin de tout un détour pour échapper aux prises de la raison, semble un acquis dans l'arabesque, laquelle ne s'embarrasse pas de ces scrupules par rapport à une logique mais est naturellement dissidente, jubilatoire, érotique.

\section{Conclusion : œcuménisme esthétique}

Les invariants ornementaux méditerranéens semblent répondre à un sens commun esthétique, cette capacité d'apprécier le beau s'appuyant sur un consentement préalable et objet d'une satisfaction désintéressée qu'on qualifie depuis Kant de goût. Le fait que ce goût soit universellement communicable sans médiation d'un concept fait qu'il puisse s'abreuver tant d'art académique que d'arts appliqués, voire de créations 
des artisans autodidactes, depuis les plus renommés, tels Tahar Ben Jelloun, consacré peintre depuis peu, jusqu'à ces artistes en retrait, tels Isravele, anagramme de " elevarsi " (s'élever), cet ermite et mosaïste outsider, «irrégulier ", maçon de son état, retiré depuis vingt ans sur le monte Gallo sur les hauteurs de Palerme dans une tour de guet militaire de l'époque bourbonne après un songe, une vision d'un ange qui porte des jumeaux dans ses bras : le Christ et lui-même. Son sanctuaire (Figures 1 et 2 : sanctuaire d'Isravele, photos de l'auteur, mai 2015) ou plutôt le chef-d'œuvre de ce "facteur Cheval» palermitain, réunit dans son panthéon des décors en mandorle où les symboles de la chrétienté (agneau, anges) côtoient ceux de l'islam (main de Fatima), de l'hébraïsme (dont l'étoile de David qu'il aurait composée par hasard en emboîtant deux triangles), du maçonnisme (équerre), de l'imagerie populaire (cœurs), du bouddhisme (mandalas), voire les images votives de piété (Padre Pio), ainsi qu'une mythologie personnelle onirique et fantasmatique. Nous avons là autant de métastases formelles qui foisonnent selon un mouvement centripète (Isravele ramasse des morceaux de terre cuite, de carreaux de faïence, de culs de bouteilles, de coquillages) et centrifuge (son sanctuaire, telle une nouvelle « chapelle palatine » des rois normands, rayonne de mille tesselles).

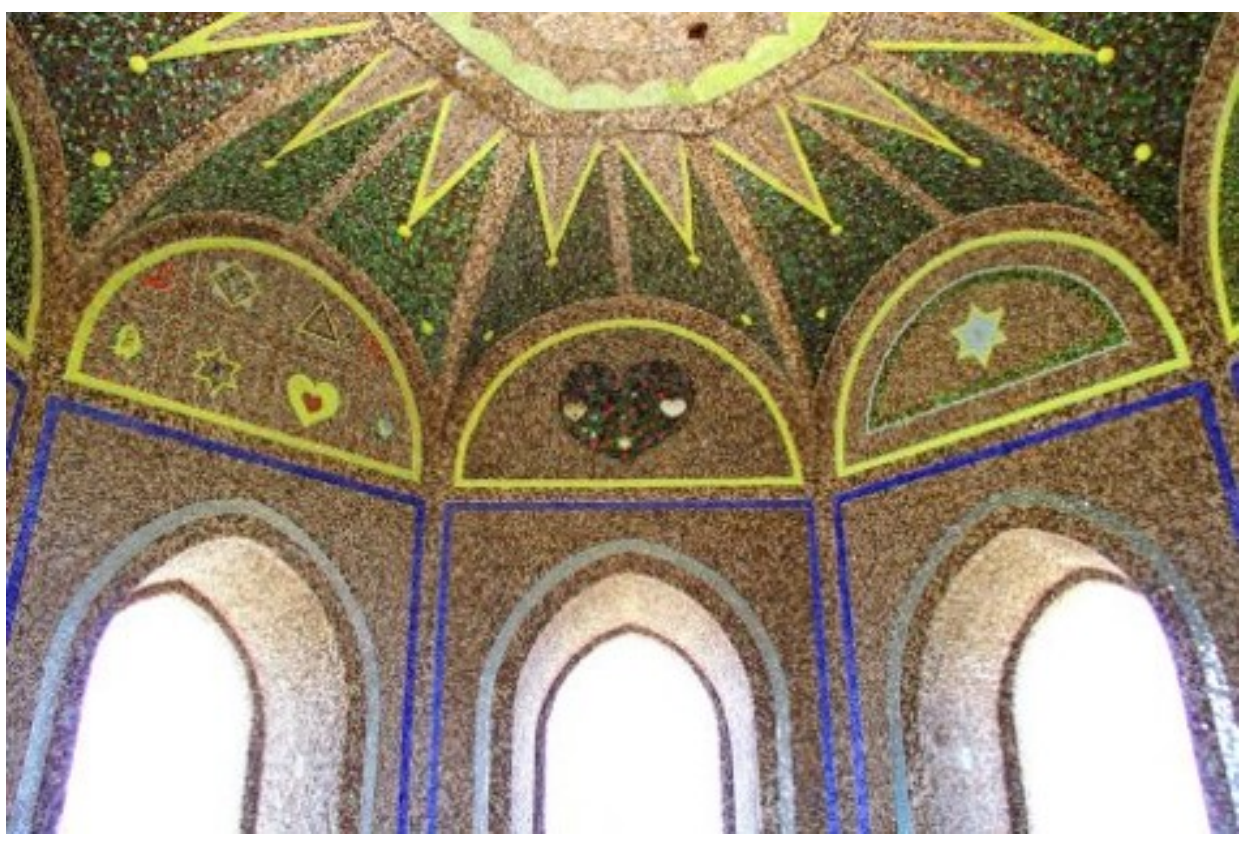




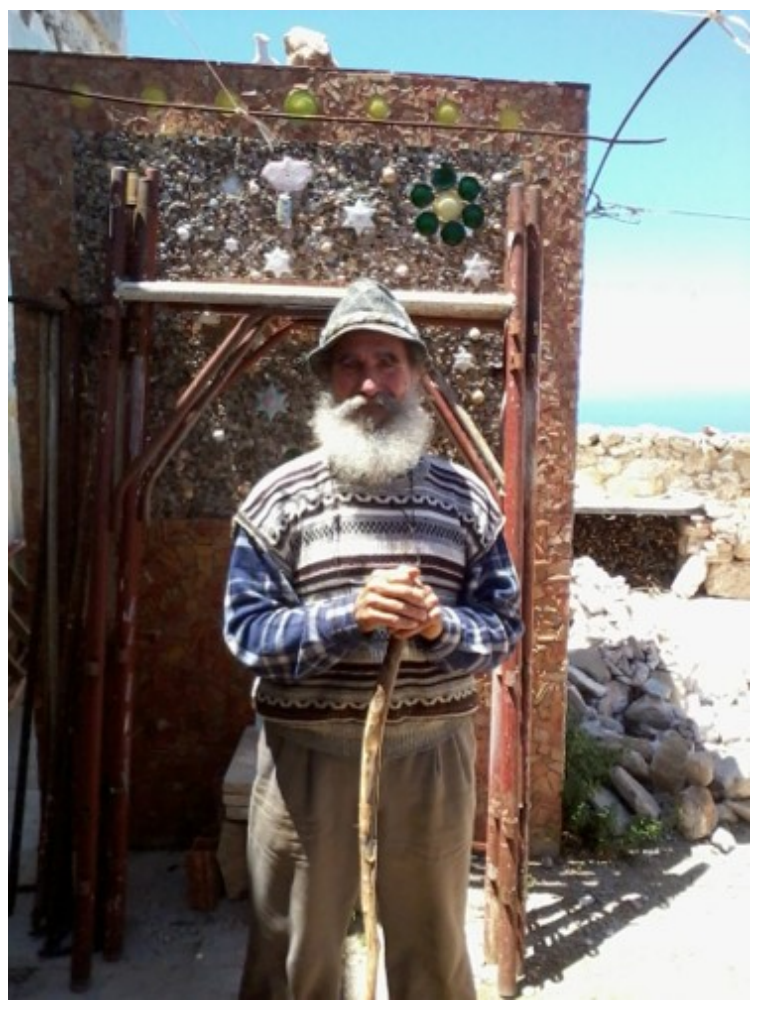

S’impose encore une fois la métaphore du "bricolage", voire du "rapiéçage » mémoriel, à partir de matériaux de récupération qui peuvent être empruntés à différentes cultures pourvu qu'ils s'enchâssent dans la forme enfantée. Du haut de son observatoire, Isravele jouit en outre d'une ubiquité paisible et réalise pour ainsi dire cette symbiose entre Orient et Occident, Europe et Afrique, s'emparant de la transhumance de motifs et leur conférant une aura inédite provoquant le ravissement. Il célèbre à sa façon la beauté d'un patrimoine à la fois tangible et intangible commun, cette pulsion humaine vers le décoratif, au-delà des débats entre ligne et couleur, des polarités entre artisanat et art académique, des clivages entre profane et religieux, dévotion et irrévérence, dans une énergétique, un embrasement, une force d'expansion, transcendant toute frontière nationale ou confessionnelle.

\section{BIBLIOGRAPHIE}

ARAGON Louis (1926), Le Paysan de Paris, Paris : Gallimard.

BATAILlE Georges (1980), Lascaux ou la naissance de l'art, Genève : Skira.

BARTHES Roland (1957) « Le vin et le lait », Mythologies, Paris : Seuil.

BARTHES Roland (1973), Le plaisir du texte, Paris : Seuil.

BARTHES Roland (1975), « De l'œuvre au texte », Essais Critiques IV [1971], Paris : Seuil, 75-76. 
BEN JELLOUN Tahar (1992), L'ange aveugle, Paris : Gallimard.

BEN JELLOUN Tahar (2013), Lettre à Matisse, et autres écrits sur l'art, Paris : Gallimard.

BEN JELLOUN Tahar (2017), J'essaie de peindre la lumière du monde, Paris : Gallimard / Institut du Monde Arabe.

BONNE Jean-Claude, DENOYELLE Martine, MICHEL Christian, NOUVEL-KAMMERER Odile \& COQUERY Emmanuel (2010), « Y a-t-il une lecture symbolique de l'ornement ? », Perspective (1), 27-42. BRILLAT-SAVARIN Anthelme (1981), Physiologie du goût [1826], Paris : Herman.

CHEVREUL Eugène (1939), De la loi du contraste simultané des couleurs [1839], Paris : Pitois-Levrault. DELEUZE Gilles (1984), Logique de la sensation, Paris : La Différence.

FAURE Elie (1992), L'esprit des formes [1923], Paris : Gallimard.

GOMBRICH Ernst (1971), L'art et l'illusion. Psychologie de la représentation picturale [1960], Paris :

Gallimard.

GROUPE MU (1992), Traité du signe visuel. Pour une rhétorique de l'image, Paris : Seuil.

KHATIBI Abdelkébir \& Sijelmassi Mohamed (1994), L'art calligraphique de l'Islam, Paris : Gallimard.

KHATIBI Abdelkébir (2006), Pèlerinage d'un artiste amoureux, Paris : Le Serpent à plumes.

LE CORBUSIER (1996), L'art décoratif aujourd'hui [1925], Paris : Flammarion.

LÉVI-STRAUSS Claude (1962), La pensée sauvage, Paris : Plon.

LOTMAN Youri (1992), La sémiosphère, Limoges : PULIM.

LYOTARD Jean-François (1971), Discours, Figure, Paris : Klincksieck.

MALDINEY Henry (2012), Regard, Parole, Espace, Paris : Cerf.

MILLET Laurence (2002), L'ABCdaire de Matisse, Paris : Flammarion.

OURSEl Hervé (1986), Peintures et dessins du Musée Pouchkine et du Musée de l'Ermitage, Lille : Musée des beaux-Arts.

SERRES, Michel (1985), Les cinq sens, Paris : Grasset.

VALÉRY Paul (1924), L'âme et la danse in Eupalinos ou l'architecte, Paris : Gallimard.

VON UEXKÜLL Jakob (2010), Milieu animal et milieu humain [1956], Paris : Payot \& Rivages.

\section{RÉSUMÉS}

Le constat d'une transhumance de motifs ornementaux autour du bassin méditerranéen nous a incités à avancer l'hypothèse d'un œcuménisme esthétique. Le transfert nord-sud se joue entre Henri Matisse et Tahar Ben Jelloun. Le séjour marocain du premier lui fit dépasser le fauvisme vers une stylisation pré-mimétique et un chromatisme incandescent qui sert de source d'inspiration pour son homologue en herbe lequel, en retour, revisite les broderies et tapis tissés par des mains de femmes illettrées de son enfance. Le transfert est-ouest se vérifie lorsque la découverte de la ville troglodytique en terrasses de Matera rappelle à Ben Jelloun la médina de Fès dont les souks bariolés l'enjoignent à « habiller » la pierre monochrome de couleurs vives et imaginées. Ces croisements sont possibles dès lors que le motif s'affranchit du visible, tel le figural de Jean-François Lyotard, lesté de désir et que, par la pulsation des formes, une «énergie 
spatialisante » (Maldiney) en émane. Pour Abdelkébir Khatibi, la plénitude du sacré de l'art islamique irradie en effet tant dans l'architecture que dans les arts appliqués. C'est l'occasion pour nous d'appréhender les rives de la Méditerranée comme dépositaires de motifs qui migrent de la peinture à l'artisanat ou à la gastronomie, faisant de la synesthésie non plus une figure de style mais une puissance polysensorielle qui met le spectateur en immersion, au-delà de toute frontière confessionnelle ou nationale.

The evidence of ornamental transhumance around the Mediterranean basin encouraged us to propose the hypothesis of an aesthetic ecumenism. The north-south transfer occurs between Henri Matisse and Tahar Ben Jelloun. The Moroccan journey of the former helped him to evolve from fauvism into pre-mimetic stylization and incandescent chromatism, which on his side inspired his homologue revisiting the embroideries and tapestries woven by the illiterate women's hands of his youth. The east-west transfer emerges when the troglodyte city in terraces of Matera reminds Ben Jelloun of the Fès' medina whose varicoloured souks urge him to "dress" the monochrome stone with vivid and imaginary colours. These crossings are possible since the motive emancipates from the visible, in the wake of Jean-François Lyotards "figural", charged with desire and, due to the pulsation of forms, generates "spatialising energy" (Maldiney). According to Abdelkébir Khatibi, in Islamic arts the fullness of the sacred irradiates in both architecture and applied arts. It gives us the opportunity to reflect on the Mediterranean shores as depository of motives and colours which migrate from painting to craftwork or to gastronomy, turning synaesthesia into a polysensorial potency putting the beholder into immersion, beyond any confessional and national border.

\section{INDEX}

Mots-clés : motifs ornementaux, syncrétisme, œcuménisme, Matisse, Ben Jelloun

Keywords : ornamental motives, syncretism, ecumenism, Matisse, Ben Jelloun

\section{AUTEUR}

\section{NATHALIE ROELENS}

Université du Luxembourg - Institut d'Études Romanes, Médias et Arts (IRMA) 